\section{TATRA \\ MOUNTaiNS \\ Mathematical Publications}

DOI: $10.2478 / \mathrm{tmmp}-2013-0012$

Tatra Mt. Math. Publ. 54 (2013), 153-163

\title{
THE EXISTENCE OF MULTIPLE SOLUTIONS FOR BOUNDARY VALUE PROBLEM WITH ONE DIMENSIONAL P-LAPLACIAN
}

\author{
BORIS RUdOLF
}

\begin{abstract}
The boundary value problem for a differential equation with one dimensional p-Laplacian is studied. The technique of lower and upper solutions is used. The existence of a solution for well ordered and unordered case as well as the existence of multiple solutions are proved. The growth condition is assumed only on a part of the nonlinearity.
\end{abstract}

The paper deals with the boundary value problem

$$
\begin{gathered}
\left(\varphi_{p}\left(x^{\prime}\right)\right)^{\prime}=F\left(t, x, \varphi_{p}\left(x^{\prime}\right)\right), \\
x(b)=\int_{0}^{b} x(s) d g(s)-k \varphi_{p}\left(x^{\prime}(b)\right), \quad x^{\prime}(0)=0 .
\end{gathered}
$$

The left hand side of (1) is one dimensional p-Laplacian, $\varphi_{p}(s)=|s|^{p-1} \operatorname{sgn}(s)$, the right hand side is the sum of two continuous functions

$$
F(t, x, y)=f_{1}(t, x, y)+f_{2}(t, x, y)
$$

with different properties. The second boundary condition is nonlocal, $g(s)$ is a nondecreasing function of bounded variation, $G(s)=\operatorname{var}_{[0, s]} g(\tau), G(b)<1$ and $k \geq 0$.

The nonlocal boundary condition covers certain types of linear two point, multipoint and integral boundary conditions.

The main goal is to prove existence of multiple classical solutions of (1), (2), i.e., solutions from

$$
D=\left\{x \in C^{1}(I), \varphi_{p}\left(x^{\prime}\right) \in C^{1}(I)\right\}, \quad I=[0, b]
$$

under suitable conditions.

(C) 2013 Mathematical Institute, Slovak Academy of Sciences. 2010 Mathematics Subject Classification: 34B10, $34 \mathrm{~B} 15$.

Keywords: p-Laplacian, nonlocal boundary condition, lower and upper solution.

Supported by grant 1/0021/10 of the Scientific Grant Agency VEGA of Slovak Republic. 


\section{BORIS RUDOLF}

We use the method of lower and upper solutions. Results of this type for the classical second order BVP are well known, see [4], [7], existence results for periodic BVP with p-Laplacian and growth condition on nonlinearity are in [5], and existence result for (1), (2) in well ordered case and restricted growth of nonlinearity is given in [8].

The paper is based on ideas of [7] applied on the problem (1), (2). By a priori estimates of the norm of solutions or its derivatives we use method of $\mathrm{Kor}$ m a n [3], based on B i h a ri inequality [1].

We define a lower and upper solution of (1), (2) by the obvious way. Set $I^{0}=I \backslash\left\{t_{i} ; 0<t_{i}<b, i=1 \ldots n\right\}$.

Definition 1 ([8]). A function $\alpha \in C(I) \cup C^{1}\left(I^{0}\right)$, with $\varphi\left(\alpha^{\prime}\right) \in C^{1}\left(I^{0}\right)$ is called a lower solution of (1), (2) if

$$
\begin{array}{rlrl}
\lim _{t \rightarrow t_{i}-} \alpha^{\prime}(t) & \leq \lim _{t \rightarrow t_{i}+} \alpha^{\prime}(t) & \text { for } \quad i=1, \ldots, n, \\
\left(\varphi_{p}\left(\alpha^{\prime}(t)\right)\right)^{\prime} & \geq f\left(t, \alpha(t), \varphi_{p}\left(\alpha^{\prime}(t)\right)\right) & \text { for } \quad t \in I^{0}, \\
\alpha(b) & \leq \int_{0}^{b} \alpha(s) d g(s)-k \varphi_{p}\left(\alpha^{\prime}(b)\right), & & \alpha^{\prime}(0) \geq 0 .
\end{array}
$$

Similarly a function $\beta \in C(I) \cup C^{1}\left(I^{0}\right)$, with $\varphi\left(\beta^{\prime}\right) \in C^{1}\left(I^{0}\right)$ is called an upper solution of $(1),(2)$ if

$$
\begin{array}{rlrl}
\lim _{t \rightarrow t_{i}-} \beta^{\prime}(t) & \geq \lim _{t \rightarrow t_{i}+} \beta^{\prime}(t) & & \text { for } \quad i=1, \ldots, n, \\
\left(\varphi_{p}\left(\beta^{\prime}(t)\right)\right)^{\prime} & \leq f\left(t, \beta(t), \varphi_{p}\left(\beta^{\prime}(t)\right)\right) & \text { for } \quad t \in I^{0}, \\
\beta(b) & \geq \int_{0}^{b} \beta(s) d g(s)-k \varphi_{p}\left(\beta^{\prime}(b)\right), & & \beta^{\prime}(0) \leq 0 .
\end{array}
$$

In the case of strict inequalities for the equation on $I^{0}$ and for the second boundary condition we say that lower and upper solutions are strict.

LEMMA 2 ([8]). Let $\alpha, \beta$ be a strict lower and upper solution and $x$ be a solution of the problem (1), (2). Then $\alpha(t) \leq x(t)$ for each $t \in I$ implies $\alpha(t)<x(t)$ for each $t \in I$ and $\beta(t) \geq x(t)$ for each $t \in I$ implies $\beta(t)>x(t)$ for each $t \in I$.

In our first existence result as well as in all theorems below we assume that the summand $f_{1}$ satisfies a Nagumo-Bernstein type of growth condition [2], [5] and $f_{2}$ instead of this satisfies one of two possible types of sign condition.

In the rest of the paper constant $q>0$ is such that $\frac{1}{p}+\frac{1}{q}=1$. Then the function $\varphi_{q}$ is the inverse of $\varphi_{p}$. 


\section{MULTIPLE SOLUTIONS FOR BVP WITH P-LAPLACIAN}

Theorem 3. Let

(A1) $\exists r>0$ such that $\forall t \in I, f_{1}(t, r, 0)>0, f_{1}(t,-r, 0)<0$ on $I$,

(B1) $\exists c>0$ such that $\forall t \in I,|x|<r, y \in R$,

$$
\left|f_{1}(t, x, y)\right| \leq c\left(1+|y|^{q}\right),
$$

(C1) $\forall t \in I, x, y \in R$,

$$
x \cdot f_{2}(t, x, y) \geq 0 .
$$

Then the problem (1), (2) possesses a solution $x$ such that $|x(t)|<r$.

Proof. Set $X=C^{1}([0, b])$ and define an operator $T: X \rightarrow X$ by

$$
T x(t)=\frac{1}{G(b)-1}\left\{\int_{0}^{b} G(s) \varphi_{q}\left(\tilde{F}_{x}(s)\right) d s+k\left(\tilde{F}_{x}(b)\right)\right\}-\int_{t}^{b} \varphi_{q}\left(\tilde{F}_{x}(s)\right) d s,
$$

where

Then

$$
\tilde{F}_{x}(s)=\int_{0}^{s} F\left(\tau, x(\tau), \varphi_{p}\left(x^{\prime}(\tau)\right)\right) d \tau .
$$

$$
T x(t) \in D=\left\{x \in C^{1}(I), \varphi_{p}\left(x^{\prime}\right) \in C^{1}(I)\right\}, \quad \text { and } \quad(T x)^{\prime}(0)=0 .
$$

The function satisfies boundary conditions (2), see [8], and fixed point of completely continuous operator $T$ is a solution of (1), (2).

We consider the perturbed boundary value problems

with $\lambda \in[0,1]$.

$$
\begin{aligned}
& \left(\varphi_{p}\left(x^{\prime}\right)\right)^{\prime}=\lambda F\left(t, x, \varphi_{p}\left(x^{\prime}\right)\right)+(1-\lambda) x(t), \\
& x(b)=\int_{0}^{b} x(s) d g(s)-k \varphi_{p}\left(x^{\prime}(b)\right), \quad x^{\prime}(0)=0
\end{aligned}
$$

Clearly, $-r$ and $r$ are strict lower and upper solutions of (4), (5).

Convex combination $f_{1 \lambda}=\lambda f_{1}+(1-\lambda) x$ satisfies the same growth condition (A1) with the constant $c_{1}=c+r$ and $f_{2 \lambda}=\lambda f_{2}$ satisfies the sign condition (C1)independently on $\lambda$.

Let $x(t)$ be a solution of (4), (5) with $|x(t)|<r$ on $I$. We estimate $\left|x^{\prime}(t)\right|$.

Suppose $x\left(t_{0}\right)=\max _{\left[t_{0}, t_{1}\right]}, x(t)>0$ and $x^{\prime}(t)<0$ on $\left(t_{0}, t_{1}\right)$. Then $x(t)$ is invertible on $\left[t_{0}, t_{1}\right]$, its inverse is $t(x)$. Denote $p(x)=\varphi_{p}\left(x^{\prime}(t(x))\right)$.

We rewrite the equation (4) in

$$
p^{\prime}(x) \varphi_{q}(p(x))=f_{1 \lambda}(t, x, p(x))+f_{2 \lambda}(t, x, p(x))
$$

and estimate

$$
p^{\prime}(x) \varphi_{q}(p(x)) \geq-c_{1}\left(1+|p(x)|^{q}\right) .
$$




\section{BORIS RUDOLF}

Substitution $z(x)=|p(x)|^{q}$ gives

$$
z^{\prime}(x) \geq-q c_{1}(1+z(x))
$$

and after the change of variable $v=x_{0}-x$ we denote $\tilde{z}(v)=z(x)+1$ and obtain

$$
\tilde{z}^{\prime}(v) \leq q c_{1} \tilde{z}(v), \quad \tilde{z}(0)=1 .
$$

Then

$$
\tilde{z}(v) \leq e^{q c_{1} v} \leq e^{q c_{1} r} \quad \text { and } \quad|p(x)| \leq e^{c_{1} r}=c_{2} .
$$

That means, $-\varphi_{q}\left(c_{2}\right) \leq x^{\prime}(t) \leq 0$ on $\left[t_{0}, t_{1}\right]$. Boundedness of derivative in case $x>0$ and $x^{\prime}>0$ and in cases $x<0$ is proved similarly. Then boundedness of a solution $\mathrm{x}, x(t)<r$ implies the existence of a constant $\rho, x^{\prime}(t)<\rho$ on $I$.

We set

$$
\Omega_{r, \rho}=\left\{x \in X ;|x|<r,\left|x^{\prime}\right|<\rho\right\} .
$$

The associated homotopy operator

$$
H(x, \lambda)=\frac{1}{G(b)-1}\left\{\int_{0}^{b} G(s) \varphi_{q}\left(\tilde{F}_{x, \lambda}(s)\right) d s+k\left(\tilde{F}_{x, \lambda}(b)\right)\right\}-\int_{t}^{b} \varphi_{q}\left(\tilde{F}_{x, \lambda}(s)\right) d s,
$$

with $\tilde{F}_{x, \lambda}(s)=\int_{0}^{s} \lambda f_{1}\left(\tau, x(\tau), \varphi_{p}\left(x^{\prime}(\tau)\right)\right)+f_{2}\left(\tau, x(\tau), \varphi_{p}\left(x^{\prime}(\tau)\right)\right)+(1-\lambda) x(\tau) d \tau$ possesses no fixed point on the boundary of $\Omega_{r, \rho}$. Then the Leray-Schauder degree of $H(., \lambda)$ is well defined and independent on $\lambda$.

$H(x, 0)$ is an odd operator and Borsuk's theorem implies [2]

$$
d(I-T, \Omega, 0)=d(I-H(x, 0), \Omega, 0)=1(\bmod 2)
$$

which implies the existence of a fixed point $x \in \Omega$ of $T$.

By the same method can be proved the following existence result with another sign condition.

Theorem 4. Let

(A1) $\exists r>0$ such that $\forall t \in I, f_{1}(t, r, 0)>0, f_{1}(t,-r, 0)<0$ on $I$,

(B1) $\exists c>0$ such that $\forall t \in I,|x|<r, y \in R$,

$$
\left|f_{1}(t, x, y)\right| \leq c\left(1+|y|^{q}\right),
$$

(C2) $\forall t \in I, x, y \in R$,

$$
y \cdot f_{2}(t, x, y) \leq 0 .
$$

Then the problem (1), (2) possesses a solution $x$ such that $|x(t)|<r$.

In the case of well ordered nonconstant lower and upper solutions the following theorem holds. 


\section{MULTIPLE SOLUTIONS FOR BVP WITH P-LAPLACIAN}

TheOREM 5. Let

(A2) $\alpha(t) \leq \beta(t)$ be a lower and upper solution of (1), (2),

(B2) $\exists c(r): R^{+} \rightarrow R^{+}$such that $\forall t \in I,|x|<r, y \in R$,

$$
\left|f_{1}(t, x, y)\right| \leq c(r)\left(1+|y|^{q}\right),
$$

(C1) or (C2).

Then the problem (1), (2) possesses a solution $x$ such that $\alpha(t) \leq x(t) \leq \beta(t)$.

P r o of. We prove the case when (C1) holds. Set $r=\max \{\|\alpha\|,\|\beta\|\}+1$, and choose $M>\max \{|f(t, x, 0)| ; t \in I, \alpha(t) \leq x \leq \beta(t)\}$.

Define

$$
f_{1}^{*}(t, x, y)= \begin{cases}f_{1}(t, \beta(t), y)+M(r-\beta(t)), & x>r \\ f_{1}(t, \beta(t), y)+M(x-\beta(t)), & \beta(t)<x \leq r, \\ f_{1}(t, x, y), & \alpha(t) \leq x \leq \beta(t), \\ f_{1}(t, \alpha(t), y)-M(\alpha(t)-x), & -r \leq x<\alpha(t), \\ f_{1}(t, \alpha(t), y)-M(\alpha(t)+r), & x<-r,\end{cases}
$$

and

$$
f_{2}^{*}(t, x, y)= \begin{cases}f_{2}(t, \beta(t), y), & 0 \leq \beta(t)<x, \\ f_{2}(t, \beta(t), y) \frac{x}{\beta(t)}, & \beta(t)<x<0, \\ 0, & \beta(t)<0 \leq x, \\ f_{2}(t, x, y), & \alpha(t) \leq x \leq \beta(t), \\ 0, & x \leq \alpha(t)<0, \\ f_{2}(t, \alpha(t), y) \frac{x}{\alpha(t)}, & 0<x<\alpha(t), \\ f_{2}(t, \alpha(t), y), & x<\alpha(t) \leq 0 .\end{cases}
$$

Now the boundary value problem

$$
\begin{aligned}
& \left(\varphi_{p}\left(x^{\prime}\right)\right)^{\prime}=f_{1}^{*}\left(t, x, \varphi_{p}\left(x^{\prime}\right)\right)+f_{2}^{*}\left(t, x, \varphi_{p}\left(x^{\prime}\right)\right), \\
& x(b)=\int_{0}^{b} x(s) d g(s)-k \varphi_{p}\left(x^{\prime}(b)\right), \quad x^{\prime}(0)=0
\end{aligned}
$$

satisfies conditions (A1), (B1), and (C1). That means, perturbed BVP is solvable and

$$
d\left(I-T^{*}, \Omega_{r, \rho}, 0\right)=1(\bmod 2) .
$$

Moreover, for each $\epsilon>0$ the function $\alpha(t)-\epsilon$ is a strict lower solution and $\beta(t)+\epsilon$ is a strict upper solution of $(6),(7)$.

Lemma 2 implies that $\alpha(t) \leq x(t) \leq \beta(t)$. That means, $f_{i}^{*}\left(t, x, \varphi_{p}\left(x^{\prime}\right)\right)=$ $f_{i}\left(t, x, \varphi_{p}\left(x^{\prime}\right)\right)$ and $x(t)$ is also a solution of (1), (2). 


\section{BORIS RUDOLF}

EXAMPLE 6. We consider the boundary value problem

$$
\begin{gathered}
\left(\varphi_{p}\left(x^{\prime}\right)\right)^{\prime}+k(t, x) \varphi_{m}\left(x^{\prime}\right)+f(t, x)=h(t), \\
x(1)=\int_{0}^{1} x(s) d g(s)-k \varphi_{p}\left(x^{\prime}(1)\right), \quad x^{\prime}(0)=0,
\end{gathered}
$$

and assume that

$$
k(t, x) \geq 0, \quad m>1, \quad \text { and } \quad \lim _{x \rightarrow \infty} f(t, x)=-\infty, \quad \lim _{x \rightarrow-\infty} f(t, x)=\infty .
$$

Then BVP (8) possesses a solution for each $h(t) \in C(I)$.

EXAMPLE 7. Consider the boundary value problem

$$
\begin{gathered}
\left(\varphi_{p}\left(x^{\prime}\right)\right)^{\prime}+k(t, x) \varphi_{r}\left(x^{\prime}\right)=f\left(t, x, x^{\prime}\right), \\
x(1)=\int_{0}^{1} x(s) d g(s)-k \varphi_{p}\left(x^{\prime}(1)\right), \quad x^{\prime}(0)=0
\end{gathered}
$$

with the bounded nonlinearity $|f(t, x, y)| \leq M$, and $k(t, x) \geq 0$. Then BVP (9) possesses a solution $x(t)$,

$$
|x(t)| \leq \frac{1}{1-G(1)}\left(B_{0}+k M q^{1-p}\right)+\beta_{0}(t),
$$

where

$$
\beta_{0}(t)=M q^{1-p}\left(1-t^{q}\right) \quad \text { and } \quad B_{0}=\int_{0}^{1} \beta(s) d g(s) .
$$

The case of unordered lower and upper solutions is more complicated, requires stronger growth conditions on $f_{1}$. The existence result presented below gives no a priori bound of a solution, instead of this we have only a partial information about its localization.

Theorem 8. Let

(A3) $\alpha \not \leq \beta$ be a strict lower and upper solution of (1), (2),

(B3) $\exists 0<A, B<q-1, c>0$ such that $\forall t \in I, x, y \in R$,

$$
\left|f_{1}(t, x, y)\right| \leq c\left(1+|x|^{A}+|y|^{B}\right)
$$

(C1) or (C2).

Then the problem (1), (2) possesses a solution $x$ such that $\beta \not \leq x \not \leq \alpha$. 


\section{MULTIPLE SOLUTIONS FOR BVP WITH P-LAPLACIAN}

Proof. We set $r_{0}=\max (|\alpha|,|\beta|)$, a number $r>r_{0}$, we specify later, and $M_{0}=c\left(1+r^{A}\right)$. We define a perturbation

$$
f_{1}^{*}(t, x, y)= \begin{cases}f_{1}(t, r, y)+M_{0}, & x>r+1, \\ f_{1}(t, r, y)+M_{0}(x-r), & r<x \leq r+1, \\ f_{1}(t, x, y), & -r \leq x \leq r, \\ f_{1}(t, r, y)+M_{0}(x+r), & -r-1 \leq x<-r, \\ f_{1}(t, r, y)-M_{0}, & x<-r-1 .\end{cases}
$$

Clearly, $-r-1, \alpha, \beta, r+1$ are strict lower and upper solutions of the perturbed BVP

$$
\begin{aligned}
& \left(\varphi_{p}\left(x^{\prime}\right)\right)^{\prime}=f_{1}^{*}\left(t, x, \varphi_{p}\left(x^{\prime}\right)\right)+f_{2}\left(t, x, \varphi_{p}\left(x^{\prime}\right)\right), \\
& x(b)=\int_{0}^{b} x(s) d g(s)-k \varphi_{p}\left(x^{\prime}(b)\right), \quad x^{\prime}(0)=0 .
\end{aligned}
$$

Moreover, $f_{1}^{*}$ satisfies the growth property (B1) and (B2) for chosen $r$

$$
\left|f_{1}^{*}(t, x, y)\right| \leq c\left(1+|r|^{A}+|y|^{B}\right)+M_{0}<\bar{c}\left(1+|y|^{q}\right) .
$$

The existence theorems above and their proofs imply $\left(T^{*}\right.$ is given by $(3)$ using $f_{1}^{*}$ instead of $f$ )

$d\left(I-T^{*}, \Omega_{r+1, \rho}, 0\right)=d\left(I-T^{*}, \Omega_{\alpha, r+1, \rho}, 0\right)=d\left(I-T^{*}, \Omega_{-r-1, \beta, \rho}, 0\right)=1(\bmod 2)$. Then

$$
d\left(I-T^{*}, \Omega, 0\right)=1(\bmod 2) \quad \text { for } \Omega=\Omega_{r+1, \rho} \backslash \overline{\Omega_{\alpha, r+1, \rho} \cup \Omega_{-r-1, \beta, \rho}} .
$$

We estimate the norm of a solution $x$ using a method of K or man [3], [7]. We consider the case (C1) only.

Suppose that $\max |x(t)|=x\left(t_{0}\right)>r_{0}$ and set $M=x\left(t_{0}\right)-r_{0}>0$. The second boundary condition implies $t_{0} \neq b$. As $x \in \Omega, \exists t_{\alpha}$ such that $x\left(t_{\alpha}\right) \leq \alpha\left(t_{\alpha}\right) \leq r_{0}$. Then $\exists t_{1}$ such that $x\left(t_{1}\right)=r_{0}$.

We suppose $t_{0}<t_{1}$, the opposite case is treated similarly.

As in the proof of Theorem 3, we denote $p(x)=\varphi_{p}\left(x^{\prime}(t(x))\right), z(x)=|p(x)|^{q}$ and $v=M+r_{0}-x$, to obtain

$$
\tilde{z}^{\prime}(v) \leq q c\left(1+(r+1)^{A}+\tilde{z}(v)^{\frac{B}{q}}\right), \quad \tilde{z}(0)=0 .
$$

The B i h ar i lemma [1] implies

$$
\tilde{z}(v) \leq\left[\left(c q M\left(1+(r+1)^{A}\right)\right)^{1-\frac{B}{q}}+(q-B) c M\right]^{\frac{1}{1-\frac{B}{q}}} .
$$


Then

$$
|p(x)| \leq\left[\left(c q M\left(1+(r+1)^{A}\right)\right)^{1-\frac{B}{q}}+(q-B) c M\right]^{\frac{1}{q-B}}
$$

and

$$
|p(x)| \leq d\left(\left(c q M\left(1+(r+1)^{A}\right)\right)^{\frac{1}{q}}+((q-B) c M)^{\frac{1}{q-B}}\right), \quad d=2^{\frac{1}{q-B}} .
$$

As $M \leq(b-a) \max _{v \in[0, M]}|p(v)|$, we have

$$
M \leq d(b-a)\left(\left(c q M\left(1+(r+1)^{A}\right)\right)^{\frac{1}{q}}+((q-B) c M)^{\frac{1}{q-B}}\right) .
$$

Then

$$
M^{\frac{q-1}{q}} \leq c_{1} r^{\frac{A}{q}}+c_{2} M^{\frac{b}{(q-B) q}},
$$

where positive constants $c_{i}$ are independent on $r$.

Either

$$
r_{0}+M<r \quad \text { or } \quad r_{0}+M \geq r
$$

In the latter case

$$
M^{\frac{q-1}{q}} \leq c_{1}\left(r_{0}+M\right)^{\frac{A}{q}}+c_{2} M^{\frac{b}{(q-B) q}} .
$$

Then $\exists M_{1}$ independent of $r$ such that $r_{0}+M<r$.

We proceed similarly also in the case $\max |x(t)|=-x\left(t_{0}\right)>r_{0}$. That means, $|x(t)|<r$ and then $x$ is a solution of the original problem.

We prove the existence of at least two solutions under assumption that the boundary value problem has either two strict lower solutions and one upper one or vice versa.

Lemma 9. Let $\alpha$ be a strict lower solution of (1), (2).

Suppose (C1) holds and sets

$$
f_{1 \alpha}(t, x, y)= \begin{cases}f_{1}(t, x, y) & \text { for } t \in I, x \geq \alpha(t), y \in R, \\ f_{1}(t, \alpha(t), y) & \text { for } t \in I, x<\alpha(t), y \in R\end{cases}
$$

and

$$
f_{2 \alpha}(t, x, y)= \begin{cases}f_{2}(t, x, y) & \text { for } t \in I, x \geq \alpha(t), y \in R, \\ x f_{2}(t, \alpha(t), y) & \text { for } t \in I, 0 \leq x<\alpha(t), y \in R, \\ 0 & \text { for } t \in I, x<0 \leq \alpha(t), y \in R, \\ f_{2}(t, \alpha(t), y) & \text { for } t \in I, x<\alpha(t)<0, y \in R .\end{cases}
$$




\section{MULTIPLE SOLUTIONS FOR BVP WITH P-LAPLACIAN}

Then each solution $x(t)$ of the problem

is a solution of (1), (2).

$$
\begin{gathered}
\left(\varphi_{p}\left(x^{\prime}\right)\right)^{\prime}=F_{\alpha}\left(t, x, \varphi_{p}\left(x^{\prime}\right)\right), \\
x(b)=\int_{0}^{b} x(s) d g(s)-k \varphi_{p}\left(x^{\prime}(b)\right), \quad x^{\prime}(0)=0
\end{gathered}
$$

Proof. The proof is based on the fact that in both cases the function $\alpha(t)-m$ is a strict lower solution of (14) for each $m>0$. Then Lemma 2 implies our assertion.

Theorem 10. Let

(A4) $\alpha \leq \beta, \alpha \leq \alpha_{1}, \alpha_{1} \not \leq \beta, \alpha, \alpha_{1}$ be a strict lower and $\beta$ a strict upper solution of (1), (2),

(B4) $\exists 0<A, B<q-1, c>0$ such that $\forall t \in I, x, y \in R$,

(B2), (C1).

$$
f_{1}(t, x, y) \geq-c\left(1+|x|^{A}+|y|^{B}\right),
$$

Then the boundary value problem (1), (2) possesses at least two solutions.

Proof. As $\alpha \leq \beta$, are well ordered lower and upper solutions, assumptions (A2), (B2), (C1) and Theorem 2 implies the existence of a solution $x_{1}$.

On the other hand, $\alpha_{1} \not \leq \beta$, are unordered lower and upper solutions of (1), (2) and also of (14). Moreover, each solution $x_{2}$ of (14) is $x_{2} \geq \alpha$.

We modify (14) replacing $f_{1 \alpha}$ by one sided perturbation

$$
f_{1 \alpha}^{*}= \begin{cases}f_{1 \alpha}(t, r, y)+M_{0}, & x>r+1, \\ f_{1 \alpha}(t, r, y)+M_{0}(x-r), & r<x \leq r+1, \\ f_{1 \alpha}(t, x, y), & x \leq r,\end{cases}
$$

where $r, M_{0}$ have the same sense as in the proof of Theorem 8 .

It is easy to prove by the same method as in the case of Theorem 8 that $x(t)<r$ for each solution $x(t)$ of modified problem. Then there exists a second solution $x_{2}$ such that $\alpha<x_{2}, \beta \not \leq x_{2} \not \leq \alpha_{1}$.

In the case when solution are bounded from above by the strict upper solution $\beta$ the following symmetric lemma and multiplicity theorem hold.

LEMMA 11. Let $\beta$ be a strict upper solution of (1), (2).

Suppose (C1) holds and sets

$$
f_{1 \beta}(t, x, y)= \begin{cases}f_{1}(t, x, y) & \text { for } t \in I, x \leq \beta(t), y \in R \\ f_{1}(t, \beta(t), y) & \text { for } t \in I, x>\beta(t), y \in R\end{cases}
$$

and 


\section{BORIS RUDOLF}

$$
f_{2 \beta}(t, x, y)= \begin{cases}f_{2}(t, x, y) & \text { for } t \in I, x \leq \beta(t), y \in R, \\ x f_{2}(t, \beta(t), y) & \text { for } t \in I, 0 \geq x<\beta(t), y \in R, \\ 0 & \text { for } t \in I, x>0 \geq \beta(t), y \in R, \\ f_{2}(t, \beta(t), y) & \text { for } t \in I, x>\beta(t)>0, y \in R .\end{cases}
$$

Then each solution $x(t)$ of the problem

$$
\begin{gathered}
\left(\varphi_{p}\left(x^{\prime}\right)\right)^{\prime}=F_{\beta}\left(t, x, \varphi_{p}\left(x^{\prime}\right)\right) \\
x(b)=\int_{0}^{b} x(s) d g(s)-k \varphi_{p}\left(x^{\prime}(b)\right), \quad x^{\prime}(0)=0
\end{gathered}
$$

is a solution of (1), (2).

\section{TheOrem 12. Let}

(A4) $\alpha \leq \beta, \beta_{1} \leq \beta, \alpha \leq \beta_{1}, \alpha$ be a strict lower and $\beta \beta_{1}$ a strict upper solutions of $(1),(2)$,

(B4) $\exists 0<A, B<q-1, c>0$ such that $\forall t \in I, x, y \in R$,

$$
f_{1}(t, x, y) \geq-c\left(1+|x|^{A}+|y|^{B}\right)
$$

(B2), (C1).

Then the boundary value problem (1), (2) possesses at least two solutions.

EXAMPLE 13. We consider the boundary value problem

$$
\begin{gathered}
\left(\varphi_{p}\left(x^{\prime}\right)\right)^{\prime}-k(t, x) \varphi_{m}(x)+f(t, x)=h(t), \\
x(1)=\int_{0}^{1} x(s) d g(s)-k \varphi_{p}\left(x^{\prime}(1)\right), \quad x^{\prime}(0)=0,
\end{gathered}
$$

where we assume that $k(t, x) \geq 0$ for $t \in I, x, y \in R$ and the function $f$ is such that

$$
\lim _{x \rightarrow-\infty} f(t, x)=\infty, \quad \lim _{x \rightarrow \infty} f(t, x)=-\infty
$$

and

$$
\exists x_{1}, x_{2}, x_{1}<x_{2} \text { such that } f\left(t, x_{1}\right)<f\left(t, x_{2}\right) \text { on } I .
$$

Then the boundary value problem (18) has a solution for each $h(t)$, at least two solutions for

$$
h(t), f\left(t, x_{1}\right) \leq h(t) \leq f\left(t, x_{2}\right) \text { on } I,
$$

and at least three solutions for

$$
h(t), f\left(t, x_{1}\right)<h(t)<f\left(t, x_{2}\right) \text { on } I .
$$




\section{MULTIPLE SOLUTIONS FOR BVP WITH P-LAPLACIAN}

\section{REFERENCES}

[1] BIHARI, I.: A generalization of a lemma of Bellman and its applications to uniqueness problems of differential equations, Acta Math. Acad. Sci. Hung. 7 (1956), 81-94.

[2] GAINES, R. E.-MAWHIN J.: Coincidence Degree, and Nonlinear Differential Equations, in: Lecture Notes in Math., Vol. 568, Springer, New York, 1977.

[3] KORMAN, PH.: Remarks on Nagumo's condition, Portugal. Math. 55 (1998), 1-9.

[4] RACHŮNKOVÁ, I.: On the existence of two solutions of the periodic problem for the ordinary second order differential equation, Nonlinear Anal. 22 (1994), 1315-1322.

[5] RACHƯNKOVÁ, I.-STANĚK, S.-TVRDÝ, M.: Singularities and Laplacians in Boundary Value Problems for Nonlinear Ordinary Differential Equations, in: Handbook of Differential Equations, Vol. 3, Elsevier, New York, 2006, pp. 607-723.

[6] RUDOLF, B.: Method of lower and upper solutions for a generalized boundary value problem, Arch. Math. (Brno) 36 (2000), 595-602.

[7] RUDOLF, B.: An existence and multiplicity result for a periodic boundary value problem, Math. Bohem. 133 (2008), 41-61.

[8] RUDOLF, B.: On a boundary value problem for differential equation with p-Laplacian, Tatra Mt. Math. Publ. 48 (2011), 189-195.

Received August 8, 2012

Department of Mathematics FEI STU

Mlynská dolina

SK-812-19 Bratislava

SLOVAKIA

E-mail: boris.rudolf@stuba.sk 